\title{
Integral Input-to-State Stability of Networked Control Systems
}

\author{
Navid Noroozi ${ }^{\circledR}$, Roman Geiselhart ${ }^{\circledR}$, Seyed Hossein Mousavi ${ }^{\circledR}$, Romain Postoyan ${ }^{\circledR}$, \\ and Fabian R. Wirth (1)
}

\begin{abstract}
We investigate integral input-to-state stability (iISS) of nonlinear networked control systems (NCSs). The controller is designed by emulation, i.e., it is constructed to ensure iISS for the closed-loop system in the absence of the network. Afterward, the latter is taken into account and explicit conditions are provided on the scheduling protocol and the maximum allowable transmission interval to preserve iISS for the NCS. The results are applied to two case studies: bilinear systems and neutrally stable linear systems under saturated feedback, where the conditions are formulated as linear matrix inequalities. The effectiveness of the results is further illustrated via a numerical example.
\end{abstract}

Index Terms-Hybrid systems, integral input-to-state stability (iISS), Lyapunov methods, networked control systems (NCSs).

\section{INTRODUCTION}

In networked control systems (NCSs), both sensor and actuator data are transmitted over a digital channel. The recent interest in NCSs is motivated by the many benefits they offer, such as the ease of maintenance and installation, configuration flexibility, reduced weight and volume, and lower cost compared to wired point-to-point connections. In order to exploit the full potential of this emerging technology, novel design and analysis approaches are needed, to ensure control functionality despite the communication imperfections induced by the network.

A popular design approach for NCSs is the so-called emulation method, see, e.g., [1]-[5] and references therein. The idea is to first ignore communication constraints and design a continuous-time controller for the continuous-time plant. Then, the controller is implemented via the network and it is shown that the stability property of the

Manuscript received July 4, 2018; revised December 15, 2018 and May 19, 2019; accepted June 11, 2019. Date of publication June 27, 2019; date of current version February 27, 2020. The work of N. Noroozi was partially supported by the Alexander von Humboldt Foundation. The work of R. Postoyan was supported by ANR via grant HANDY (ANR-18-CE400010). Recommended by Associate Editor F. Mazenc. (Corresponding author: Navid Noroozi.)

N. Noroozi is with the Laboratory for Systems Theory and Automatic Control, Otto-von-Guericke University Magdeburg, Magdeburg 39106, Germany (e-mail: navid.noroozi@ovgu.de).

R. Geiselhart is with the Institute of Measurement, Control and Microtechnology, University of Ulm, Ulm 89081, Germany (e-mail: roman. geiselhart@uni-ulm.de).

S. H. Mousavi is with the University Health Network, University of Toronto, Toronto, ON M5G 2A2, Canada (e-mail: s.h.mousavi65@ gmail.com).

R. Postoyan is with the CNRS, CRAN, Université de Lorraine, Nancy F-54000, France (e-mail: romain.postoyan @ univ-lorraine.fr).

F. R. Wirth is with the Faculty of Computer Science and Mathematics, University of Passau, Passau 94032, Germany (e-mail: fabian. lastname@uni-passau.de).

Color versions of one or more of the figures in this paper are available online at http://ieeexplore.ieee.org.

Digital Object Identifier 10.1109/TAC.2019.2925449 closed-loop continuous-time system is preserved for the NCS under suitable conditions on the network. Typically, the maximum allowable transmission interval (MATI) needs to be sufficiently small and, when scheduling occurs, the protocol has to satisfy a certain stability property, see [2] and [6].

Since plants with disturbances are prevalent in control applications, there have been many attempts toward a stability analysis of nonlinear NCSs in the presence of disturbances via the emulation-based setting. For instance, $\mathcal{L}_{p}$ stability of NCSs is addressed in e.g., [2], [7], and [8] and input-to-state stability (ISS) of NCSs is considered in e.g., [4], [9], and [10]. In this paper, we investigate a different, yet relevant, stability notion for systems with inputs: integral input-to-state stability (iISS) [11]. This concept is of fundamental importance in control theory as it extends $H_{2}$ stability to nonlinear systems, cf., [11] and [12] for more details. Roughly speaking, iISS captures the notion that the system state remains small, regarding the initial conditions, provided that the integral of the input (i.e., the input energy) is small.

Building upon the work of [6] where the stability of NCS with no disturbances is addressed, this paper investigates iISS of NCSs subject to time-varying transmissions and scheduling. We adapt and extend the approach in [6] to ensure an iISS property for the NCS with respect to disturbances on the plant dynamics, the measurement, the control input, and the transmitted data. Inspired by the recent results in [13] on iISS of continuous-time systems, we introduce Lyapunov-based conditions permitting both additive and multiplicative disturbances. This avoids unnecessary conservatism, which may arise when attempting to upper bound the cross terms by additive terms. As a result, a less conservative upper bound on the MATI, compared to the case where the cross terms are upper bounded by additive terms, is obtained. While the results of [6] have been already generalized to the case of ISS, e.g., [4], [10], and [14], iISS is a more general property, which requires a different set of assumptions and different proof techniques. For instance, in comparison to conditions in [4], [10], and [14], the gain function governing the decay of the associated iISS Lyapunov function does not have to be monotonic. Last but not least, unlike most of the current literature, e.g., [2], [4], [7], and [14], we not only consider measurement noise but also disturbances induced by the network, which can be due to quantization errors or unmodeled transmission delays. The presence of disturbances leads to the possibility of growth of state trajectories during jumps, which renders the analysis more difficult. Our results are applied to two case studies: bilinear systems and linear system under saturated feedback, where the conditions are formulated as linear matrix inequalities. We further illustrate the results via a numerical example.

This paper expands on the conference paper [15], where integral input-to-state practical stability is mainly studied, while asymptotic properties are ensured here. In addition, the assumptions are different and more tailored to the problem at hand, and the case studies of bilinear systems and linear system under saturated feedback are provided. It can be noted that the results are also new in the sampled-data case, i.e., when no scheduling occurs. 


\section{Preliminaries}

\section{A. Notation}

$\mathbb{R}_{\geq 0}\left(\mathbb{R}_{>0}\right)$ and $\mathbb{Z}_{\geq 0}(\mathbb{N})$ are the nonnegative (strictly positive) real numbers and the nonnegative (strictly positive) integers, respectively. The standard Euclidean norm is denoted by $|\cdot|$. For a closed set $\mathcal{A} \subset \mathbb{R}^{n}$ and any point $x \in \mathbb{R}^{n}$, we denote $|x|_{\mathcal{A}}:=\min _{y \in \mathcal{A}}|x-y|$. For any pair $(x, y) \in \mathbb{R}^{n} \times \mathbb{R}^{m}$, we write $(x, y)$ to represent $\left[x^{\top}, y^{\top}\right]^{\top}$. We will consider $\mathcal{K}, \mathcal{K}_{\infty}$, and $\mathcal{K} \mathcal{L}$ comparison functions, see [16, Ch. 4.4] for definitions. A function $\beta: \mathbb{R}_{\geq 0} \times \mathbb{R}_{\geq 0} \times \mathbb{R}_{\geq 0} \rightarrow \mathbb{R}_{\geq 0}$ is of class $\mathcal{K} \mathcal{L} \mathcal{L}$ $(\beta \in \mathcal{K} \mathcal{L} \mathcal{L})$, if for each $s \geq 0, \beta(\cdot, s, \cdot) \in \mathcal{K} \mathcal{L}$ and $\beta(\cdot, \cdot, s) \in \mathcal{K} \mathcal{L}$. The identity function is denoted by id.

\section{B. Hybrid Systems}

Consider a hybrid system with state $x \in \mathbb{R}^{n}$ and input $w \in \mathbb{R}^{d}$ defined by

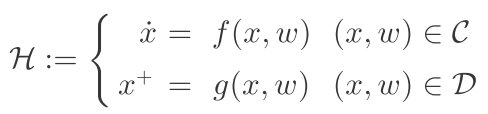

where $\mathcal{C}$ and $\mathcal{D}$ are closed subsets of $\mathbb{R}^{n} \times \mathbb{R}^{d}, f: \mathcal{C} \rightarrow \mathbb{R}^{n}$ and $g$ : $\mathcal{D} \rightarrow \mathbb{R}^{n}$ are continuous. We refer to $\mathcal{C}$ and $\mathcal{D}$ as the flow set and the jump set, cf., [17] and [18].

We recall the following definitions concerning hybrid time domains from [17] and [18]. A subset $E \subset \mathbb{R}_{>0} \times \mathbb{Z}_{>0}$ is called a hybrid time domain if for all $(T, J) \in E, E \cap([0, T] \times\{0,1, \ldots, J\})=$ $\bigcup_{j=0}^{J}\left(\left[t_{j}, t_{j+1}\right], j\right)$ for some finite sequence of real numbers $0=t_{0} \leq$ $\leq t_{J+1}$. For each hybrid time domain $E$, there is a lexicographic ordering of points: given $(t, j),\left(t^{\prime}, j^{\prime}\right) \in E$, we say $(t, j) \preceq\left(t^{\prime}, j^{\prime}\right)$ if $t+j \leq t^{\prime}+j^{\prime}$, and $(t, j) \prec\left(t^{\prime}, j^{\prime}\right)$ if $t+j<t^{\prime}+j^{\prime}$. For $s \in \mathbb{R}$, $i(s):=\sup \left\{i \in \mathbb{Z}_{\geq 0}:(s, i) \in E\right\}$ whenever the set on the right-hand side is nonempty.

A function defined on a hybrid time domain $E$ is called a hybrid signal. A hybrid signal $x$ is called a hybrid arc if for each $j \in \mathbb{Z}_{>0}$, the function $x(\cdot, j)$ is locally absolutely continuous on the interval $I_{j}:=$ $\{t \in \mathbb{R}:(t, j) \in E\}$. For the hybrid arc $x$, we denote $x_{0}:=x(0,0)$. A hybrid signal $w: E \rightarrow \mathbb{R}^{d}$ is called a hybrid input if for each $j \in \mathbb{Z}_{\geq 0}$, $w(\cdot, j)$ is Lebesgue-measurable and locally essentially bounded on $I_{j}$. If $x: E \rightarrow \mathbb{R}^{n}$ is a hybrid signal, we also use the notation dom $x:=E$ instead of $E$, if we want to stress this is the domain of definition of $x$. Due to lack of space, the notion of a solution pair $(x, w)$ to $\mathcal{H}$ is not presented here, see [17] for detailed information.

\section{Problem Statement}

Consider the nonlinear plant model

$$
\begin{aligned}
\dot{x}_{p} & =f_{p}\left(x_{p}, u, d_{x}\right) \\
y & =g_{p}\left(x_{p}, d_{y}\right)
\end{aligned}
$$

where $f_{p}: \mathbb{R}^{n_{p}} \times \mathbb{R}^{n_{u}} \times \mathbb{R}^{n_{d x}} \rightarrow \mathbb{R}^{n_{p}}$ and $g_{p}: \mathbb{R}^{n_{p}} \times \mathbb{R}^{n_{d y}} \rightarrow$ $\mathbb{R}^{n_{y}}$ are continuously differentiable. Here, $x_{p} \in \mathbb{R}^{n_{p}}$ is the plant state, $u \in \mathbb{R}^{n_{u}}$ is the control input, $d_{x} \in \mathbb{R}^{n_{d x}}$ is the disturbance input, and $y \in \mathbb{R}^{n_{y}}$ is the plant output, which is affected by $d_{y} \in \mathbb{R}^{n_{d y}}$. The disturbance $d_{y}$ can model either some uncertainty in the output function or some measurement noise. We also assume that we know a continuoustime controller, which ensures that the closed-loop system with (2) is iISS with inputs $d_{x}, d_{y}$, and $d_{u}$ in the absence of a communication network. We focus on dynamic controllers of the form

$$
\begin{aligned}
\dot{x}_{c} & =f_{c}\left(x_{c}, y\right) \\
u & =g_{c}\left(x_{c}, d_{u}\right)
\end{aligned}
$$

where again $f_{c}: \mathbb{R}^{n_{c}} \times \mathbb{R}^{n_{y}} \rightarrow \mathbb{R}^{n_{c}}$ and $g_{c}: \mathbb{R}^{n_{c}} \times \mathbb{R}^{n_{d u}} \rightarrow \mathbb{R}^{n_{u}}$ are continuously differentiable. Here, $x_{c} \in \mathbb{R}^{n_{c}}$ is the controller state, and $d_{u} \in \mathbb{R}^{n_{d u}}$ is the disturbance affecting the control input. Similar motivations as those for $d_{y}$ apply to the disturbance $d_{u}$.

We consider the scenario where the plant and the controller are connected via a packet-based communication network that is composed of $\ell \in \mathbb{N}$ nodes. A node corresponds to a collection of sensors and/or actuators of the plant and the controller.

The network generates various constraints on the communication of both $u$ and $y$. In this paper, we concentrate on the effect due to time-varying sampling and scheduling. Note that we also consider some other networked-induced effects including quantization as perturbations to the system. Transmissions occur only at some given time instants $t_{j}, j \in \mathbb{N}$ satisfying $\epsilon \leq t_{j}-t_{j-1} \leq \tau_{\text {MATI }}$. Here, $\tau_{\text {MATI }}$ is a given constant representing the maximum time between any two transmission instants, whereas $\epsilon \in\left(0, \tau_{\mathrm{MATI}}\right]$ represents the minimum intertransmission time. Note that $\epsilon$ can be taken arbitrarily small and prevents Zeno behavior [18] in the hybrid model that will be derived later. Furthermore, at each transmission instant, a single node is granted access to the network. This selection is done by the scheduling protocol. As in [2], the overall system can be modeled by the following impulsive system:

$$
\begin{aligned}
\dot{x}_{p} & =f_{p}\left(x_{p}, \hat{u}, d_{x}\right) & & t \in\left[t_{j-1}, t_{j}\right] \\
y & =g_{p}\left(x_{p}, d_{y}\right) & & t \in\left[t_{j-1}, t_{j}\right] \\
\dot{x}_{c} & =f_{c}\left(x_{c}, \hat{y}\right) & & \\
u & =g_{c}\left(x_{c}, d_{u}\right) & & t \in\left[t_{j-1}, t_{j}\right] \\
\dot{\hat{y}} & =\hat{f}_{p}\left(x_{p}, x_{c}, \hat{y}, \hat{u}\right) & & t \in\left[t_{j-1}, t_{j}\right] \\
\dot{\hat{u}} & =\hat{f}_{c}\left(x_{p}, x_{c}, \hat{y}, \hat{u}\right) & & \\
\hat{y}\left(t_{j}^{+}\right) & =y\left(t_{j}\right)+h_{y}\left(j, e\left(t_{j}\right), v_{y}\left(t_{j}\right)\right) & & \\
\hat{u}\left(t_{j}^{+}\right) & =u\left(t_{j}\right)+h_{u}\left(j, e\left(t_{j}\right), v_{u}\left(t_{j}\right)\right) & & \\
x_{p}\left(t_{j}^{+}\right) & =x_{p}\left(t_{j}\right) & & \\
x_{c}\left(t_{j}^{+}\right) & =x_{c}\left(t_{j}\right) & &
\end{aligned}
$$

where $\hat{u} \in \mathbb{R}^{n_{u}}$ and $\hat{y} \in \mathbb{R}^{n_{y}}$ are, respectively, the currently available estimates of the true controller output at the plant side, and the estimate of the measurements at the controller side.

These two variables are updated at the transmission times $t_{j}, j \in \mathbb{N}$ and evolve according to the dynamics $\hat{f}_{p}$ and $\hat{f}_{c}$ between transmission instants. For instance, the use of zero-order-hold devices leads to $\hat{f}_{p}=0, \hat{f}_{c}=0$, and model-based techniques may also be envisioned, see Section V-B. The functions $h_{y}$ and $h_{u}$ accommodate the effect of the transmission protocol on the updates of $\hat{y}\left(t_{j}\right)$ and $\hat{u}\left(t_{j}\right)$, respectively, at the transmission times $t_{j}$, see [2]. The signal $v:=\left(v_{y}, v_{u}\right) \in \mathbb{R}^{n_{v}}$ denotes a disturbance corrupting the data transmitted over the network at transmission instants. The data corruption can be due to several network-induced imperfections such as the quantization effect (cf., [19] for more details). In addition, $e:=\left(e_{y}, e_{u}\right) \in \mathbb{R}^{n_{e}}$ denotes the network-induced errors where $e_{y}:=\hat{y}-y \in \mathbb{R}^{n_{y}}$ and $e_{u}:=\hat{u}-u \in \mathbb{R}^{n_{u}}$.

In order to reformulate the system in a specific form, we make the following assumptions.

Assumption 1: The disturbance $d_{x}$ is a measurable and essentially bounded function of time. The perturbation signals $d_{y}$ and $d_{u}$ in (4) are uniformly bounded and continuously differentiable functions of time and the signal $v$ is a uniformly bounded sequence. 
Given $x:=\left(x_{p}, x_{c}\right) \in \mathbb{R}^{n_{x}}, h:=\left(h_{y}, h_{u}\right) \in \mathbb{R}^{n_{e}}, w:=\left(d_{x}, d_{y}\right.$, $\left.d_{u}, \dot{d}_{y}, \dot{d}_{u}\right) \in \mathbb{R}^{n_{w}}$ with $n_{w}:=n_{d}+2 n_{u}+2 n_{y}$, we rewrite (4) as

$$
\begin{aligned}
\dot{x} & =f(x, e, w) \\
\dot{e} & =g(x, e, w) \\
e\left(t_{j}^{+}\right) & =h\left(j, e\left(t_{j}\right), v\right)
\end{aligned}
$$

where $f: \mathbb{R}^{n_{x}} \times \mathbb{R}^{n_{e}} \times \mathbb{R}^{n_{w}} \rightarrow \mathbb{R}^{n_{x}}$ and $g: \mathbb{R}^{n_{x}} \times \mathbb{R}^{n_{e}} \times \mathbb{R}^{n_{w}}$ $\rightarrow \mathbb{R}^{n_{e}}$ are defined by $f(x, e, w):=\left(f_{p}\left(x_{p}, g_{c}\left(x_{c}, d_{u}\right)+e_{u}, d_{x}\right)\right.$, $\left.f_{c}\left(x_{c}, g_{p}\left(x_{p}, d_{y}\right)+e_{y}\right)\right), \quad g(x, e, w):=\left(\hat{f}_{p}\left(x_{p}, x_{c}, g_{p}\left(x_{p}, d_{y}\right)+\right.\right.$ $\left.e_{y}, g_{c}\left(x_{c}, d_{u}\right)+e_{u}, d_{x}\right)-\frac{\partial g_{p}}{\partial x_{p}}\left(x_{p}, d_{y}\right) f_{p}\left(x_{p}, g_{c}\left(x_{c}, d_{u}\right)+e_{u}, d_{x}\right)$ $-\frac{\partial g_{p}}{\partial d_{y}}\left(x_{p}, d_{y}\right) \dot{d}_{y}, \hat{f}_{c}\left(x_{p}, x_{c}, g_{p}\left(x_{p}, d_{y}\right)+e_{y}, g_{c}\left(x_{c}, d_{u}\right)+e_{u}, d_{x}\right)$ $\left.-\frac{\partial g_{c}}{\partial x_{c}}\left(x_{c}, d_{u}\right) f_{c}\left(x_{c}, g_{p}\left(x_{p}, d_{y}\right)+e_{y}\right)-\frac{\partial g_{c}}{\partial d_{u}}\left(x_{c}, d_{u}\right) \dot{d}_{u}\right)$. The function $h$ in (5c) is called the scheduling protocol. In the absence of $v$, the precise description of $h$ for a large class of standard protocols including round-robin (RR), try-once-discard (TOD), and sampled-data (SD) protocols is studied in [2]. In the presence of the disturbances, this particular description of $h$ may be given by

$$
e\left(t_{j}^{+}\right)=\left(I_{n_{e}}-\Psi\left(e\left(t_{j}\right), j\right)\right) e\left(t_{j}\right)+\Psi\left(e\left(t_{j}\right), j\right) v\left(t_{j}\right) .
$$

The protocol is fully defined by the function

$$
\Psi(e, j):=\operatorname{diag}\left(\psi_{1}(e, j) I_{n_{1}}, \ldots, \psi_{\ell}(e, j) I_{n_{\ell}}\right)
$$

where $\psi_{i}, i=1, \ldots, \ell$, are mappings from $\mathbb{R}^{n_{e}} \times \mathbb{Z}_{\geq 0}$ to $\{0,1\}$ (cf., [2, Sec. III] for precise definitions of $\psi_{i}$ for RR, TOD, and SD protocols). Equation (6) describes the update of $e$ at each transmission, where only the transmitted data are influenced by the disturbance $v$ at the transmission instant.

The aim is to provide conditions on system (2), controller (3), the scheduling protocol, and an upper bound on the MATI for which iISS is guaranteed for NCS (5). Toward this end, we transform the NCS into a hybrid system in the formalism of [18]. We introduce a clock variable $\tau \in \mathbb{R}_{\geq 0}$ representing the time elapsed since the last transmission. We also introduce $\kappa \in \mathbb{Z}_{>0}$ to count the number of transmissions, which is useful to model static protocols such as RR.

In that way, we have

$$
\begin{aligned}
& \left.\begin{array}{l}
\dot{x}=f(x, e, w) \\
\dot{e}=g(x, e, w) \\
\dot{\tau}=1 \\
\dot{\kappa}=0
\end{array}\right\} \tau \in\left[0, \tau_{\text {MATI }}\right] \\
& \left.\begin{array}{l}
x^{+}=x \\
e^{+}=h(\kappa, e, v) \\
\tau^{+}=0 \\
\kappa^{+}=\kappa+1
\end{array}\right\} \tau \in\left[\epsilon, \tau_{\text {MATI }}\right] .
\end{aligned}
$$

According to (8), the flow set $\mathcal{C}$ and the jump set $\mathcal{D}$ are given by $\mathcal{C}=\left\{(x, e, \tau, \kappa, w, v): \tau \in\left[0, \tau_{\text {MATI }}\right]\right\}$ and $\mathcal{D}=\{(x, e, \tau, \kappa, w, v):$ $\left.\tau \in\left[\epsilon, \tau_{\text {MATI }}\right]\right\}$, respectively. For the sake of convenience, denote $\xi:=(x, e, \tau, \kappa), F(\xi, w, v):=(f(x, e, w), g(x, e, w), 1,0)$, and $G(\xi, w, v):=(x, h(\kappa, e, v), 0, \kappa+1)$. We can then write system (8) as

$$
\mathcal{H}:= \begin{cases}\dot{\xi}=F(\xi, w, v) & (\xi,(w, v)) \in \mathcal{C} \\ \xi^{+}=G(\xi, w, v) & (\xi,(w, v)) \in \mathcal{D}\end{cases}
$$

Let $(\xi,(w, v))$ be a solution pair to system (9). We denote its hybrid time domain by $E$.

The objective is to ensure that the set $\mathcal{A}:=\{(x, e, \tau, \kappa): x=0, e=$ $0\}$ is iISS as defined next.
Definition 2: The hybrid system (9) is said to be uniformly integral input-to-state stable (UiISS) in $\mathcal{A}$ with respect to the perturbation signals $(w, v)$ if there exist $\alpha \in \mathcal{K}_{\infty}, \eta_{1}, \eta_{2} \in \mathcal{K}$, and $\beta \in \mathcal{K} \mathcal{L} \mathcal{L}$ such that any solution pair $(\xi,(w, v))$ of (9) satisfies

$$
\begin{aligned}
& \alpha\left(|\xi(t, j)|_{\mathcal{A}}\right) \leq \beta\left(\left|\xi_{0}\right|_{\mathcal{A}}, t, j\right)+\int_{0}^{t} \eta_{1}(|w(s, i(s))|) d s \\
&+\sum_{\left(t^{\prime}, j^{\prime}\right) \in \Gamma(v)} \eta_{2}\left(\left|v\left(t^{\prime}, j^{\prime}\right)\right|\right) \\
&(0,0) \preceq\left(t^{\prime}, j^{\prime}\right) \prec(t, j)
\end{aligned}
$$

for all $(t, j) \in E$, where $\Gamma(v):=\{(t, j) \in E:(t, j+1) \in E\}$.

The estimate of the form (10) is consistent with [20], where general hybrid systems are investigated. This estimate covers the classic ones for pure continuous-time and discrete-time systems. We also note that the bound (10) is more general than the one in [15, Definition 1] as there are exogenous inputs affecting the jumps.

\section{Main REsults}

We provide a set of conditions that can be used for systems influenced by both additive and multiplicative disturbances/uncertainties. In particular, we aim at conditions that provide bounds on MATI. Inspired by the dissipation conditions in [13, Th. 14], we make the following assumption.

Assumption 3: There exist locally Lipschitz functions $V: \mathbb{R}^{n_{x}} \rightarrow$ $\mathbb{R}_{\geq 0}, W: \mathbb{Z}_{\geq 0} \times \mathbb{R}^{n_{e}} \rightarrow \mathbb{R}_{\geq 0}$, a continuous function $H: \mathbb{R}^{n_{x}} \rightarrow$ $\mathbb{R}_{\geq 0}, \underline{\alpha}_{x}, \bar{\alpha}_{x}, \underline{\alpha}_{e}, \bar{\alpha}_{e} \in \mathcal{K}_{\infty}, \varrho \in \mathcal{P} \mathcal{D}, \theta_{1}, \theta_{2}, \theta_{3}, \theta_{4}, \sigma_{1}^{V}, \sigma_{2}^{V}, \sigma_{1}^{W}, \sigma_{2}^{W}$, $\sigma_{1}, \sigma_{2}, \sigma_{v} \in \mathcal{K} \cup\{0\}$, real numbers $L, \gamma>0$, and $\lambda \in(0,1)$ such that for all $x \in \mathbb{R}^{n_{x}}$

$$
\underline{\alpha}_{x}(|x|) \leq V(x) \leq \bar{\alpha}_{x}(|x|)
$$

and for almost all $x \in \mathbb{R}^{n_{x}}$, for all $e \in \mathbb{R}^{n_{e}}$, all $\kappa \in \mathbb{Z}_{>0}$, and all $w \in \mathbb{R}^{n w}$, it holds that

$$
\begin{aligned}
& \langle\nabla V(x), f(x, e, w)\rangle \leq-\varrho(|x|)-\varrho(W(\kappa, e))+\sigma_{1}^{V}(|w|) \theta_{1}(V(x)) \\
& +\sigma_{1}^{W}(|w|) \theta_{2}(W(\kappa, e))-H^{2}(x)+\gamma^{2} W^{2}(\kappa, e)+\sigma_{1}(|w|) .
\end{aligned}
$$

In addition, it holds that

$$
\begin{aligned}
& \underline{\alpha}_{e}(|e|) \leq W(\kappa, e) \leq \bar{\alpha}_{e}(|e|) \quad \forall \kappa \in \mathbb{Z}_{\geq 0} \quad \forall e \in \mathbb{R}^{n_{e}} \\
& W(\kappa+1, h(\kappa, e, v)) \leq \lambda W(\kappa, e)+\sigma_{v}(|v|) \\
& \forall \kappa \in \mathbb{Z}_{\geq 0} \quad \forall e \in \mathbb{R}^{n_{e}} \quad \forall v \in \mathbb{R}^{n_{v}}
\end{aligned}
$$

and for almost all $e \in \mathbb{R}^{n_{e}}$, for all $x \in \mathbb{R}^{n_{x}}$, all $\kappa \in \mathbb{Z}_{\geq 0}$, and all $w \in \mathbb{R}^{n_{w}}$

$$
\begin{aligned}
& \left\langle\frac{\partial W(\kappa, e)}{\partial e}, g(x, e, w)\right\rangle \leq L W(\kappa, e)+H(x) \\
& +\sigma_{2}^{V}(|w|) \theta_{3}(V(x))+\sigma_{2}^{W}(|w|) \theta_{4}(W(\kappa, e))+\sigma_{2}(|w|) .
\end{aligned}
$$

Define $\theta(\cdot):=\max \left\{\hat{\theta}_{1}(\cdot), \hat{\theta}_{2}\left(\sqrt{\frac{\overline{\bar{\gamma}}}{\gamma}}\right)\right\}$, where $\hat{\theta}_{1}(\cdot):=2 \max \left\{\theta_{1}(\cdot)\right.$, $\left.\theta_{3}^{2}(\cdot)\right\}$ and $\hat{\theta}_{2}(\cdot):=2 \max \left\{\theta_{2}(\cdot), \operatorname{id}(\cdot) \theta_{4}(\cdot)\right\}$. The function $\theta$ satisfies the following condition:

$$
\lim _{z \rightarrow \infty} \int_{0}^{z} \frac{d r}{1+\theta(r)}=\infty
$$

Conditions (11)-(13) imply that controller (3) guarantees iISS of the origin for system $\dot{x}=f(x, e, w)$ with respect to the inputs $e$ and $w$. In particular, inequality (12) is of the form of a dissipative iISS-Lyapunov estimate for pure continuous-time systems, see [13, Definition 13]. Moreover, when the functions $\theta_{1}$ and $\theta_{2}$ in (12) are identically zero, 
inequality (12) reduces to a classic iISS-Lyapunov estimate, e.g., [12, Definition II.2]. These properties may be directly verified on the closedloop system in the absence of a digital network. Conditions (13)-(14) imply that the transmission protocol is uniformly input-to-state stable (UISS) [19]. In the absence of measurement noise, the transmission protocol is uniformly globally asymptotically stable (UGAS). As shown later (see Lemma 4), UGAS implies UISS if the function $h$ in (5c) satisfies a globally Lipschitz property in the third argument (uniformly in the first two arguments). We note that a wide range of transmission protocols such as RR, TOD, and SD are known to be UGAS and satisfy the required Lipschitz condition, in view of (6)-(7). Sufficient conditions for (15) are that $g$ is globally Lipschitz and zero at zero, and $W$ is globally Lipschitz in $e$ uniformly in $\kappa$. We also note that Assumption 3 is similar to [6, Assumption 1] while we additionally take the disturbances into account. Finally, similar to [13, Definition 13], condition (16) implies that the function $\theta$ cannot grow too quickly. The function $\theta$ is used later to scale an initial Lyapunov function candidate. Condition (16) guarantees that the resulting scaled Lyapunov function candidate is radially unbounded (cf., the proof of Theorem 5 in Appendix B for more details).

The following result shows an ISS property of the protocol $h$ used in (5c). The proof follows from a straightforward application of Lipschitz properties of $W$ and $h$, e.g., see the proof of [21, Proposition 1].

Lemma 4: Consider the discrete-time system $e(j+1)=$ $h(j, e(j), v)$ from (5c). Assume that $h$ is globally Lipschitz in the third argument, uniformly in the other arguments. Assume that for the input-free system $e(j+1)=h(j, e(j), 0)$ there exist $W: \mathbb{Z}_{\geq 0} \times \mathbb{R}^{n_{e}} \rightarrow \mathbb{R}_{\geq 0}$, which is globally Lipschitz in the second argument with constant $M$, uniformly in the first argument, functions $\underline{\alpha}_{e}, \bar{\alpha}_{e} \in \mathcal{K}_{\infty}$ and real number $\lambda \in(0,1)$ such that (13) and (14) are satisfied for $v \equiv 0$. Then, system (5c) satisfies (13) and (14) with $\sigma_{v}=M$ id.

Let $\lambda, \gamma, L$ be the constants coming from Assumption 3 and consider constants $c>1, \tilde{\lambda} \in(\lambda, 1)$. We define

$$
\tilde{\mathcal{T}}(c, \tilde{\lambda}, \gamma, L):=\left\{\begin{array}{lr}
\frac{1}{L r} \tan ^{-1}\left(\frac{r(1-\tilde{\lambda})}{2 \frac{\tilde{\lambda}}{\bar{\lambda}}\left(\frac{\gamma}{L}\left(\frac{c+1}{2}\right)-1\right)+1+\tilde{\lambda}}\right) & L<\gamma \sqrt{c} \\
\frac{1}{L}\left(\frac{1-\tilde{\lambda}^{2}}{\tilde{\lambda}^{2}+\frac{\gamma}{L}(1+c) \tilde{\lambda}+1}\right) & L=\gamma \sqrt{c} \\
\frac{1}{L r} \tanh ^{-1}\left(\frac{r(1-\tilde{\lambda})}{2 \frac{\tilde{\lambda}}{2+1}\left(\frac{\gamma}{L}\left(\frac{c+1}{2}\right)-1\right)+1+\tilde{\lambda}}\right) & L>\gamma \sqrt{c}
\end{array}\right.
$$

where $r:=\sqrt{\left|(\gamma / L)^{2}-1\right|}$. Now, we are ready to present the main result of this paper. The proof is given in Appendix B.

Theorem 5: Consider system (9) and suppose that Assumptions 1 and 3 hold. Let $\lambda, \gamma, L$ be the constants coming from Assumption 3 and let $c>1, \tilde{\lambda} \in(\lambda, 1)$. If $\tau_{\text {M ATI }} \leq \tilde{\mathcal{T}}(c, \tilde{\lambda}, \gamma, L)$, then system (9) is UiISS in $\mathcal{A}$ with respect to $(w, v)$. In particular, if $\varepsilon_{1}, \varepsilon_{2}, \varepsilon_{d} \in(0,1)$ are sufficiently small such that $1+\varepsilon_{1}+\varepsilon_{2}<c$ and $\lambda^{2}\left(1+\varepsilon_{d}\right)<\tilde{\lambda}^{2}$, then the input gains $\eta_{1}$ and $\eta_{2}$, as in (10), are given by

$$
\begin{aligned}
& \eta_{1}:=2 \sigma^{V}+2 \sigma^{W}+2 \sigma_{1}+\frac{2}{\tilde{\lambda}^{2} \varepsilon_{2}} \sigma_{2}^{2} \\
& \eta_{2}:=2 \gamma \tilde{\lambda}^{-1}\left(\frac{1}{\varepsilon_{d}}+1\right) \sigma_{v}^{2}
\end{aligned}
$$

where $\sigma^{V}:=\max \left\{\sigma_{1}^{V}, \frac{1}{\bar{\lambda}^{2} \varepsilon_{1}}\left[\sigma_{2}^{V}\right]^{2}\right\}$ and $\sigma^{W}:=\max \left\{\sigma_{1}^{W}, 2 \gamma \tilde{\lambda}^{-1} \sigma_{2}^{W}\right\}$.

Theorem 5 provides the explicit upper bound (17) for the MATI. The parameters $c$ and $\tilde{\lambda}$, respectively, in (17) accommodate the influence of $w$ and $v$. The upper bound $\tilde{\mathcal{T}}$ is strictly decreasing with respect to the first two arguments. In particular, as $c \rightarrow 1$ and $\tilde{\lambda} \rightarrow \lambda$ in (17), $\tilde{\mathcal{T}}$ tends to the following function

$$
\mathcal{T}(\lambda, L, \gamma):= \begin{cases}\frac{1}{L r} \tan ^{-1}\left(\frac{r(1-\lambda)}{2 \frac{\lambda}{\lambda+1}\left(\frac{\gamma}{L}-1\right)+1+\lambda}\right) & \gamma>L \\ \frac{1}{L}\left(\frac{1-\lambda}{1+\lambda}\right) & L=\gamma . \\ \frac{1}{L r} \tanh ^{-1}\left(\frac{r(1-\lambda)}{2 \frac{\lambda}{\lambda+1}\left(\frac{\gamma}{L}-1\right)+1+\lambda}\right) & \gamma<L\end{cases}
$$

Note that (19) is the bound developed in [6] when no uncertainties are considered. In view of (19), $c$ in (17) reduces to 1 when $w=0$ is zero. Similarly, we have $\tilde{\lambda}=\lambda$ in the absence of the noise $v$ (cf., the proof of Theorem 5 in Appendix B for more details). In the presence of uncertainties, one may choose $\tilde{\mathcal{T}}$ to be as close as possible to $\mathcal{T}$ by, respectively, taking $c$ and $\tilde{\lambda}$ sufficiently close to 1 and $\lambda$. However, this comes at the price of small $\varepsilon_{1}, \varepsilon_{2}$, and $\varepsilon_{d}$ satisfying the conditions $1+\varepsilon_{1}+\varepsilon_{2}<c$ and $\lambda^{2}\left(1+\varepsilon_{d}\right)<\tilde{\lambda}^{2}$, which in turn leads to large estimates for the gains. Therefore, in view of (18), the larger $\tau_{\text {M ATI }}$ the larger the UiISS input gains $\eta_{1}$ and $\eta_{2}$. This suggests that the optimal performance of the NCS needs a compromise between the maximum transmission time interval and the UiISS input gains.

Theorem 5 makes use of Assumption 3 in which conditions (12) and (15) include both additive and multiplicative disturbances. This enlarges the applicability of the result as we recover classic iISS conditions in [15] by only considering the additive terms. Given certain choices for the multiplicative terms, our results are useful for bilinear systems (see Section V-A). We present two special cases of interest in the following, which are immediate consequences of Theorem 5 .

Corollary 6: Consider system (9) and suppose that Assumptions 1 and 3 hold without any cross terms (i.e., $\theta_{1}=\theta_{2}=\theta_{3}=\theta_{4} \equiv 0$ ). Let $\lambda, \gamma, L$ be the constants coming from Assumption 3 and let $c>1$, $\tilde{\lambda} \in(\lambda, 1)$. If $\tau_{\text {M ATI }} \leq \tilde{\mathcal{T}}(c, \tilde{\lambda}, \gamma, L)$, then system (9) is UiISS in $\mathcal{A}$ with respect to $(w, v)$. If $\varepsilon_{2}, \varepsilon_{d} \in(0,1)$ are sufficiently small such that $1+\varepsilon_{2}<c$ and $\lambda^{2}\left(1+\varepsilon_{d}\right)<\tilde{\lambda}^{2}$, then the input gains $\eta_{1}$ and $\eta_{2}$, as in (10), are given by $\eta_{1}:=2 \sigma_{1}+\frac{2}{\tilde{\lambda}^{2} \varepsilon_{2}} \sigma_{2}^{2}, \eta_{2}:=2 \gamma \tilde{\lambda}^{-1}\left(\frac{1}{\varepsilon_{d}}+1\right) \sigma_{v}^{2}$.

Note that, by setting $\theta_{1}=\theta_{2} \equiv 0$, conditions (11) and (12) also imply that the subsystem $\dot{x}=f(x, e, w)$ is $L_{2}$-gain stable from $\left(W, \sqrt{\sigma_{1}(|w|)}\right)$ to $H$ (cf., [2] and [6] for more details).

Corollary 7: Consider system (9) and let Assumption 1 hold. Suppose also that Assumption 3 holds with $\theta_{1}(s)=\theta_{4}(s)=s, \theta_{2}(s)=0$, $\theta_{3}(s)=\sqrt{s}$ for all $s>0$. If $\tau_{\text {MATI }} \leq \tilde{\mathcal{T}}(c, \tilde{\lambda}, \gamma, L)$, then system (9) is UiISS. If $\varepsilon_{1}, \varepsilon_{2}, \varepsilon_{d} \in(0,1)$ are such that $1+\varepsilon_{1}+\varepsilon_{2}<c$ and $\lambda^{2}\left(1+\varepsilon_{d}\right)<\tilde{\lambda}^{2}$, then the input gains $\eta_{1}$ and $\eta_{2}$ can be chosen as in (18).

Remark 8: It is of particular interest to use the SD protocol, where all network nodes are updated simultaneously. In this case, $(5 \mathrm{c})$ reduces to $e\left(t_{j}^{+}\right)=v$, where $v$ is the disturbance. In that way, condition (14) holds with $\lambda=0$, i.e., $W(\kappa+1, v) \leq \sigma_{v}(|v|)$ and the upper bound (19) simplifies to $\mathcal{T}(0, L, \gamma)$. The condition $\lambda^{2}\left(1+\varepsilon_{d}\right)<\tilde{\lambda}^{2}$ in Theorem 5 is trivially satisfied and the input gain $\eta_{2}$ reduces to $2 \gamma \tilde{\lambda}^{-1} \sigma_{v}^{2}$.

\section{Case Studies}

\section{A. Bilinear Systems}

In this section, we apply the results of Section IV to bilinear systems subject to both additive and multiplicative disturbances. Consider the following plant model:

$$
\left\{\begin{aligned}
\dot{x}_{p} & =A_{p} x_{p}+B_{p} u+\left(\sum_{i=1}^{n_{d}} d_{x, i} E_{i}\right) x_{p} \\
y & =C_{p} x_{p}+\left(\sum_{i=1}^{n_{y}} d_{y, i} D_{i}\right) x_{p}
\end{aligned}\right.
$$


where $d_{x, i} \in \mathbb{R}$ (respectively, $d_{y_{i}} \in \mathbb{R}$ ) denotes the $i$ th component of the vector $d_{x}$ (respectively, $d_{y}$ ) and the real matrices $A_{p}, E_{i} \in \mathbb{R}^{n_{p} \times n_{p}}$, $B_{p} \in \mathbb{R}^{n_{p} \times n_{u}}$, and $C_{p}, D_{i} \in \mathbb{R}^{n_{y} \times n_{p}}$. We assume that $\left(A_{p}, B_{p}\right)$ is stabilizable and $\left(A_{p}, C_{p}\right)$ is detectable. The plant is controlled by the following feedback law:

$$
\left\{\begin{aligned}
\dot{x}_{c} & =A_{c} x_{c}+B_{c} y \\
u & =C_{c} x_{c}+D_{c} y+d_{u}
\end{aligned}\right.
$$

where $d_{u} \in \mathbb{R}^{n_{u}}$ is the controller-to-actuator noise/disturbance and the real matrices $A_{c} \in \mathbb{R}^{n_{c} \times n_{c}}, B_{c} \in \mathbb{R}^{n_{c} \times n_{y}}, C_{p} \in \mathbb{R}^{n_{u} \times n_{c}}, D_{c} \in$ $\mathbb{R}^{n_{u} \times n_{y}}$. Controller (21) is designed to globally exponentially stabilize the origin of (20)-(21) in the absence of disturbances, which is possible as the pairs $\left(A_{p}, B_{p}\right)$ and $\left(A_{p}, C_{p}\right)$ are stabilizable and detectable, respectively. The origin of the nominal closed-loop system (i.e., when the matrices $D_{i}$ and $E_{i}$ and the measurement noise $d_{u}$ are zero) is globally exponentially stable. The closed-loop system (20)-(21) is a special class of bilinear systems whose iISS is investigated in [11]. Due to the multiplicative disturbance terms on the right-hand side of (20), the solution $x$ may grow exponentially for sufficiently large disturbances.

We assume that only the sensor data are transmitted over the communication network. Using zero-order-hold devices and following Section III, the resulting NCS is described by

$$
\begin{aligned}
& \dot{x}=\mathbf{A} x+\mathbf{B} e+\left(\sum_{i=1}^{n_{w}} w_{i} \mathbf{E}_{i}\right) x+\mathbf{E} w \\
& \dot{e}=\mathbf{C} x+\mathbf{D} e+\left(\sum_{i=1}^{n_{w}} w_{i} \mathbf{F}_{i}^{x}\right) x+\left(\sum_{i=1}^{n_{w}} w_{i} \mathbf{F}_{i}^{e}\right) e+\mathbf{F} w
\end{aligned}
$$

$$
e\left(t_{j}^{+}\right)=h(j, e, v)
$$

where $x=\left(x_{p}, x_{c}\right), w_{i} \in \mathbb{R}$ denotes the $i$ th component of the vector $w:=\left(d_{x}, d_{y}, d_{u}, \dot{d}_{y}\right), e=\hat{y}-y$ is the sensor-to-controller error, $\mathbf{A}, \mathbf{B}, \mathbf{C}$, and $\mathbf{D}$ are

$$
\begin{aligned}
& \mathbf{A}:=\left(\begin{array}{cc}
A_{p}+B_{p} D_{c} C_{p} & B_{p} C_{c} \\
B_{c} C_{p} & A_{c}
\end{array}\right), \quad \mathbf{B}:=\left(\begin{array}{c}
B_{p} D_{c} \\
B_{c}
\end{array}\right) \\
& \mathbf{C}:=\left(-C_{p}\left(A_{p}+B_{p} D_{c} C_{p}\right)-C_{p} B_{p} C_{c}\right), \quad \mathbf{D}:=-C_{p} B_{p} D_{c} .
\end{aligned}
$$

The matrices $\mathbf{E}_{i}, \mathbf{E}, \mathbf{F}_{i}^{x}, \mathbf{F}_{i}^{e}$, and $\mathbf{F}$ follow from (20) and (21). Note that matrix $\mathbf{A}$ is Hurwitz.

We show that one can use Corollary 7 to conclude the UiISS property of the system. In the sequel, we check the conditions in Assumption 3 for two different protocols: RR and TOD. From [2], in the absence of disturbances at jumps, both protocols satisfy conditions (13) and (14) with their respective functions $W$, where $\underline{\alpha}_{e}=\mathrm{id}$ and $\left|\frac{\partial W(\kappa, e)}{\partial e}\right| \leq M$ for any $\kappa \in \mathbb{Z}_{\geq 0}$, almost all $e \in \mathbb{R}^{n_{e}}$, with $M=\sqrt{\ell}$ for the RR and $M=1$ for the TOD. Moreover, $\lambda=\sqrt{(\ell-1) / \ell}$ for both protocols. In the presence of disturbances, Lemma 4 guarantees that the same functions $W$ with the same associated gain functions satisfy (13) and (14). We note that the function $\sigma_{v}=M$ id also appears on the right-hand side of (14).

To verify conditions (12) and (15) of Assumption 3, we take the quadratic function $V(x)=x^{\top} \mathbf{P} x$, where $\mathbf{P}$ is a positive definite matrix, and note that $\left\langle\frac{\partial W(\kappa, e)}{\partial e}, g(x, e, w)\right\rangle \leq\left|\frac{\partial W(\kappa, e)}{\partial e}\right||| \mathbf{C} x|+| \mathbf{D}|| e \mid+$ $\left.\left|\sum_{i=1}^{n_{w}} w_{i} \mathbf{F}_{i}^{x}\left\|x|+| \sum_{i=1}^{n_{w}} w_{i} \mathbf{F}_{i}^{e}\right\|\right|+|\mathbf{F}||w|\right) \leq M(|\mathbf{C} x|+$ $|\mathbf{D}| W(\kappa, e)+\frac{\left|\sum_{i=1}^{n} \mathbf{F}_{i}^{x} \| w\right|}{\sqrt{\lambda_{\min }(\mathbf{P})}} \sqrt{V(x)}+\left|\sum_{i=1}^{n_{w}} \mathbf{F}_{i}^{e} \| w\right| W(\kappa, e)+\mid \mathbf{F}$ $\| w \mid)$ for almost all $e \in \mathbb{R}^{n_{e}}$, for all $x \in \mathbb{R}^{n_{x}}$, all $\kappa \in \mathbb{Z}_{\geq 0}$, and all $w \in \mathbb{R}^{n_{w}}$. Then, condition (15) holds with $L=M|\mathbf{D}|$, $H(x)=M|\mathbf{C} x|, \quad \theta_{3}(\cdot)=\sqrt{\cdot}, \quad \theta_{4}=\mathrm{id}, \quad \sigma_{2}^{V}=\frac{M\left|\sum_{i=1}^{n_{w}} \mathbf{F}_{i}^{x}\right|}{\sqrt{\lambda_{\min }(\mathbf{P})}} \mathrm{id}$, $\sigma_{2}^{W}=M\left|\sum_{i=1}^{n_{w}} \mathbf{F}_{i}^{e}\right|$ id, and $\sigma_{2}=M|\mathbf{F}|$ id. We also have

$$
\begin{array}{r}
\langle\nabla V(x), f(x, e, w)\rangle \leq x^{\top}\left(\mathbf{P A}+\mathbf{A}^{\top} \mathbf{P}\right) x+x^{\top} \mathbf{P B} e \\
+e^{\top} \mathbf{B}^{\top} \mathbf{P} x+2\left|\sum_{i=1}^{n_{w}} \mathbf{E}_{i}\right||w| x^{\top} \mathbf{P} x+2 x^{\top} \mathbf{P E} w
\end{array}
$$

for almost all $x \in \mathbb{R}^{n_{x}}$, for all $e \in \mathbb{R}^{n_{e}}$, all $\kappa \in \mathbb{Z}_{>0}$, and all $w \in \mathbb{R}^{n_{w}}$ Consider the following linear matrix inequality (LMI):

$$
\left(\begin{array}{cc}
\mathbf{P A}+\mathbf{A}^{\top} \mathbf{P}+\varepsilon_{1} \mathbf{I}_{n_{x}}+M^{2} \mathbf{C}^{\top} \mathbf{C} & \mathbf{P B} \\
\mathbf{B}^{\top} \mathbf{P} & -\left(\gamma^{2}-\varepsilon_{2}\right) I_{n_{e}}
\end{array}\right) \leq 0
$$

with positive constants $\varepsilon_{1}$ and $\varepsilon_{2}$ chosen beforehand. LMI (25) always has a solution $\mathbf{P}=\mathbf{P}^{\top}>0$ as by using Schur complement we can pick $\gamma$ sufficiently large such that a solution $\mathbf{P}$ to (25) always exists. We note that (25) together with (24) implies that condition (12) holds with $\theta_{1}=$ id, $\sigma_{1}^{V}=2\left|\sum_{i=1}^{n_{w}} \mathbf{E}_{i}\right|$ id, $\theta_{2} \equiv 0, \sigma_{1}^{W} \equiv 0$, and $\sigma_{1}(s)=|\mathbf{E}|^{2} s^{2} / \varepsilon_{1}$ for all $s \in \mathbb{R}_{\geq 0}$. Minimizing $\gamma$ subject to (25) and computing MATI from (19), Corollary 7 gives UiISS of system (22).

Corollary 9: Consider system (22). Let the matrix $\mathbf{A}$ in (23) be Hurwitz. Let $\mathbf{P}$ be a solution of (25). Also, let $\tau_{\text {MATI }}$ satisfy (19), where $L=M|\mathbf{D}|$ and $\lambda=\sqrt{(\ell-1) / \ell}$. Then, system (22) is UiISS in $\mathcal{A}$ with respect to $(w, v)$, where $\mathcal{A}$ is as in Theorem 5 .

\section{B. Linear Systems Under Saturated Feedback}

We consider the following class of neutrally stable systems affected by actuator saturation and additive disturbances:

$$
\dot{x}_{p}=A_{p} x_{p}-B_{p} \sigma(u)+d_{x}
$$

where $x_{p} \in \mathbb{R}^{n_{p}}, u \in \mathbb{R}^{n_{u}}$, and the function $s \mapsto \sigma(s)=\left(\sigma_{1}(s), \ldots\right.$, $\left.\sigma_{n_{u}}(s)\right)$, which is defined by $\sigma_{i}(s):=\min \{1, m|s|\} \operatorname{sign}(s)$ for each $s \in \mathbb{R}, i=1, \ldots, n_{u}$, and $m>0$ represents the actuator saturation. We assume that $A_{p}$ is a skew-symmetric matrix and $\left(A_{p}, B_{p}\right)$ is controllable. This class of systems has been widely studied in the literature and includes physical systems such as harmonic oscillators, e.g., see [22] and [23]. As shown in [23], the control law $u=B_{p}^{\top} x_{p}$ renders the closed-loop system iISS in the absence of network.

Assume that only the sensor data are transmitted over the communication network. The variable $\hat{y}$ corresponds to $\hat{x}_{p}$ here since we assume that the full state $x_{p}$ is measured. We use the following model-based holding function to generate $\hat{x}_{p}$ between two successive transmission instants:

$$
\dot{\hat{x}}_{p}=A_{p} \hat{x}_{p}-B_{p} \sigma(u) .
$$

Following Section III, we model the resulting NCS as a hybrid system. Similar to Section V-A, we can consider RR and TOD protocols. As discussed earlier, for both protocols conditions (13) and (14) hold. Note that only an additive disturbance appears on the right-hand side of (26). Hence, we only need to verify conditions (12) and (15) of Assumption 3 when $\theta_{i} \equiv 0$ for $i \in\{1, \ldots, 4\}$ in (12) and (15). From (26), (27), and the fact that $\left|\frac{\partial W(\kappa, e)}{\partial e}\right| \leq M$ for any $\kappa \in \mathbb{Z}_{\geq 0}$, almost all $e \in \mathbb{R}^{n_{e}}$, with $M$ as in Section V-A, we have that

$$
\left\langle\frac{\partial W(\kappa, e)}{\partial e}, g\left(x, e, d_{x}\right)\right\rangle \leq M\left|A_{p}\right||e|+M\left|d_{x}\right|
$$

meaning that (15) holds with $w=d_{x}, L=M\left|A_{p}\right|, H(x) \equiv 0$, and $\sigma_{2}=M$ id. Inspired by [23, Th. 2], we take the function $V(x)=$ $\frac{1}{3}\left((1+\tilde{V}(x))^{1 / 3}-1\right)$, where $\tilde{V}(x):=\frac{c}{3}|x|^{3}+x^{\top} P x$ with $P:=$ 

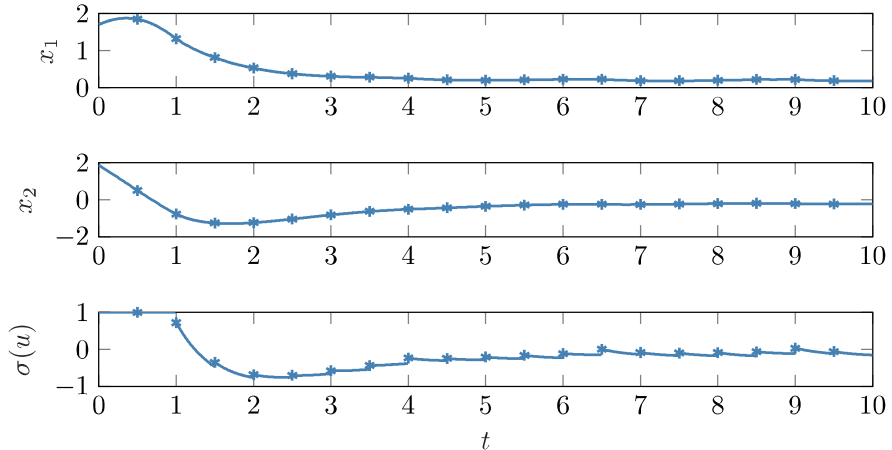

Fig. 1. State and saturated control input for $\mathcal{T}=0.5$ and the TOD protocol. $*$ denotes the transmission instants.

$P_{0}+\frac{1}{2}\left|P_{0} B_{p}\right| I$ and $c=2 m\left|P B_{p}\right|$. The matrix $P_{0}$ is any symmetric positive definite matrix such that

$$
\left(A_{p}-2 m B_{p} B_{p}^{\top}\right)^{\top} P_{0}+P_{0}\left(A_{p}-2 m B_{p} B_{p}^{\top}\right) \leq-2 I
$$

as ensured by the controllability of $\left(A_{p}, B_{p}\right)$ [23, Corollary 4]. Then, we have that

$$
\begin{aligned}
\left\langle\nabla V(x), f\left(x, e, d_{x}\right)\right\rangle \leq & \frac{-\left(1-\varepsilon_{2}\right)|x|^{2}}{(1+\tilde{V}(x))^{2 / 3}}+\frac{\varepsilon_{1} c^{2}\left|B_{p}\right|^{2}|x|^{4}}{(1+\tilde{V}(x))^{4 / 3}} \\
& +\left(\frac{1}{4 \varepsilon_{1}}+\frac{c^{2}\left|B_{p}\right|^{2}}{4 \varepsilon_{2}}\right)|e|^{2}+c_{1}\left|d_{x}\right|
\end{aligned}
$$

where $c_{1}:=3^{2 / 3} c^{1 / 3}+2|P|\left(\frac{3}{4 c}\right)^{1 / 3}$. Denote $\varrho(|x|):=\frac{\left(1-\varepsilon_{2}\right)|x|^{2}}{(1+\tilde{V}(x))^{2 / 3}}-$ $\frac{\varepsilon_{1} c^{2}\left|B_{p}\right|^{2}|x|^{4}}{(1+\tilde{V}(x))^{4 / 3}}$. Taking $\varepsilon_{1}, \varepsilon_{1}$ sufficiently small, $\varrho(x)>0$ for all $x \in$ $\mathbb{R}^{n_{p}} \backslash\{0\}$. Adding and subtracting $\varrho(W(e, \kappa)),(12)$ is satisfied with $\gamma=\sqrt{\frac{1}{4 \varepsilon_{1}}+\frac{c^{2}\left|B_{p}\right|^{2}}{4 \varepsilon_{2}}+1-\varepsilon_{2}}$ and $\sigma_{1}=c_{1}$ id.

Corollary 10: Consider system (9) with the plant dynamics as in (26), the estimator as in (27) and $u=B_{p}^{\top} \hat{x}_{p}$. Let $\tau_{\text {MATI }}$ satisfy (19), where $L=M\left|A_{p}\right|, \lambda=\sqrt{(\ell-1) / \ell}$ and $\gamma=$ $\sqrt{\frac{1}{4 \varepsilon_{1}}+\frac{c^{2}\left|B_{p}\right|^{2}}{4 \varepsilon_{2}}+1-\varepsilon_{2}}$ with $\varepsilon_{1}, \varepsilon_{2}$, and $c$ computed as above. Then system (9) is UiISS in $\mathcal{A}$ with respect to $(w, v)$, where $\mathcal{A}$ is as in Theorem 5 .

We finally illustrate the above results with application to a harmonic oscillator. Let $A_{p}$ and $B_{p}$ in (26) be given by

$$
A_{p}=\left(\begin{array}{cc}
0 & 1 \\
-1 & 0
\end{array}\right), B_{p}=\left(\begin{array}{l}
1 \\
1
\end{array}\right)
$$

and $m=1$ in (26). Solving (28) for $P_{0}$ and choosing $\varepsilon_{1}=0.25$ and $\varepsilon_{2}=0.5$, we obtain the following upper bounds for the MATIs: $\mathcal{T}=0.0560$ for RR and $\mathcal{T}=0.0601$ for TOD. Fig. 1 depicts the state trajectories of the $x_{p}$ and the saturated control input $\sigma(u)$ with a normally distributed random disturbance $d_{x}$ with mean value 0 and variance 0.2 for $\mathcal{T}=0.5$ and the TOD protocol.

\section{CONCLUSION}

This paper has investigated iISS of nonlinear NCSs. Following the emulation approach, we have given conditions under which iISS of the original continuous-time closed-loop system is preserved for the NCS. Moreover, an explicit bound on the MATI has been provided. We have adapted and extended conditions in [6] to study iISS of nonlinear NCSs influenced by both additive and multiplicative disturbances. The conditions are particularly useful for the study of iISS for bilinear systems and linear systems under saturated feedback. The effectiveness of our results was further shown via an illustrative example.

\section{APPENDIX A}

\section{TECHNICAL LEMMAS}

We state technical lemmas that are required to give the proof of Theorem 5.

Lemma 11: Consider (17). Given $c>1, \tilde{\lambda} \in(\lambda, 1)$, and $\gamma, L>0$, denote $\tilde{\mathcal{T}}:=\tilde{\mathcal{T}}(c, \tilde{\lambda}, \gamma, L)$ and let $\phi:[0, \tilde{\mathcal{T}}] \rightarrow \mathbb{R}$ be the solution to

$$
\dot{\phi}=-2 L \phi-\gamma\left(\phi^{2}+c\right) \quad \phi(0)=\tilde{\lambda}^{-1}
$$

where $\tilde{\lambda} \in(\lambda, 1)$. Then, $\phi(\tau) \in\left[\tilde{\lambda}, \tilde{\lambda}^{-1}\right]$ for all $\tau \in[0, \tilde{\mathcal{T}}]$.

The proof of Lemma 11 follows the proof of a similar result in [6] and is omitted. The following comparisonlike lemma is a variant of [20, Lemma 9].

Lemma 12: Let $\rho \in \mathcal{P D}$, and $z: E \rightarrow \mathbb{R}$ be a hybrid arc with $z_{0} \geq$ 0 . Consider a hybrid signal $v: E \rightarrow \mathbb{R}_{\geq 0}$ such that for each $j, v(\cdot, j)$ is continuous. Furthermore, assume that the following conditions hold:

1) for almost all $t$ such that $(t, j) \in E \backslash \Gamma(z)$

$$
\dot{z}(t, j) \leq-\rho(\max \{z(t, j)+v(t, j), 0\})
$$

2) for all $(t, j-1) \in \Gamma(z)$ it holds that

$$
z(t, j) \leq z(t, j-1) .
$$

Then, there exists $\tilde{\beta} \in \mathcal{K} \mathcal{L}$ such that for all $(t, j) \in E$

$$
z(t, j) \leq \max \left\{\tilde{\beta}\left(z_{0}, t\right),|v|_{(t, j), \infty}\right\}
$$

where $v \mapsto|v|_{(t, j), \infty}$ is defined in [17].

\section{APPENDIX B \\ PROOF OF THEOREM 5}

Let the quadruple $(c, \gamma, L, \tilde{\lambda})$ generate $\phi$ via (29). Consider the following hybrid Lyapunov function as in [6], for any $\xi \in \mathcal{C} \cup \mathcal{D} \cup$ $G(\mathcal{D})$ :

$$
U(\xi):=V(x)+\gamma \phi(\tau) W^{2}(\kappa, e) .
$$

By (11) and (13) and the fact that $\phi(\tau) \in\left[\tilde{\lambda}, \tilde{\lambda}^{-1}\right]$ for all $\tau \in\left[0, \tau_{\text {M ATI }}\right]$ (cf., Lemma 11), there exist $\underline{\alpha}, \bar{\alpha} \in \mathcal{K}_{\infty}$ such that the following holds:

$$
\underline{\alpha}\left(|\xi|_{\mathcal{A}}\right) \leq U(\xi) \leq \bar{\alpha}\left(|\xi|_{\mathcal{A}}\right) \quad \forall \xi \in \mathcal{C} \cup \mathcal{D} \cup G(\mathcal{D}) .
$$

For almost all $(\xi,(w, v)) \in \mathcal{C}$, we have $\langle\nabla U(\xi), F(\xi, w, v)\rangle=$ $\langle\nabla V(x), f(x, e, w)\rangle+2 \gamma \phi(\tau) W(\kappa, e)\langle\nabla W(\kappa, e), g(x, e, w)\rangle+$ $\gamma \dot{\phi}(\tau) W^{2}(\kappa, e)$. It follows from (12), (15), and (29) that

$$
\begin{aligned}
& \langle\nabla U(\xi), F(\xi, w, v)\rangle \leq-\varrho(|x|)-\varrho(W(\kappa, e))+\sigma_{1}(|w|) \\
& -H^{2}(x)-\gamma^{2} \phi^{2}(\tau) W^{2}(\kappa, e)-(c-1) \gamma^{2} W^{2}(\kappa, e) \\
& +2 \gamma \phi(\tau) \sigma_{2}^{V}(|w|) W(\kappa, e) \theta_{3}(V(x))+2 \gamma \phi(\tau) W(\kappa, e) H(x) \\
& +2 \gamma \phi(\tau) \sigma_{2}^{W}(|w|) W(\kappa, e) \theta_{4}(W(\kappa, e)) \\
& +\sigma_{1}^{V}(|w|) \theta_{1}(V(x))+\sigma_{1}^{W}(|w|) \theta_{2}(W(\kappa, e)) .
\end{aligned}
$$

Moreover, by the fact that $\phi(\tau) \leq \tilde{\lambda}^{-1}$ for all $\tau \in\left[0, \tau_{\text {MATI }}\right]$ (cf., Lemma 11) and using Young's inequality, the following holds for any 
$\varepsilon_{1}, \varepsilon_{2}>0$

$\langle\nabla U(\xi), F(\xi, w, v)\rangle \leq-\varrho(|x|)-\varrho(W(\kappa, e))+\sigma_{1}^{V}(|w|) \theta_{1}(V(x))$

$+\frac{1}{\tilde{\lambda}^{2} \varepsilon_{1}}\left[\sigma_{2}^{V}(|w|)\right]^{2} \theta_{3}^{2}(V(x))+\sigma_{1}^{W}(|w|) \theta_{2}(W(\kappa, e))$

$+2 \gamma \tilde{\lambda}^{-1} \sigma_{2}^{W}(|w|) W(\kappa, e) \theta_{4}(W(\kappa, e))$

$-\left(c-1-\varepsilon_{1}-\varepsilon_{2}\right) \gamma^{2} W^{2}(\kappa, e)+\sigma_{1}(|w|)+\frac{1}{\tilde{\lambda}^{2} \varepsilon_{2}} \sigma_{2}^{2}(|w|)$.

Letting $\hat{\sigma}(\cdot):=\sigma_{1}(\cdot)+\frac{1}{\tilde{\lambda}^{2} \varepsilon_{2}} \sigma_{2}^{2}(\cdot)$ and recalling the definition of $\sigma^{V}(\cdot), \sigma^{W}(\cdot), \hat{\theta}_{1}(\cdot)$, and $\hat{\theta}_{2}(\cdot)$ (see the statement of Theorem 5), we have $\langle\nabla U(\xi), F(\xi, w, v)\rangle \leq \sigma^{V}(|w|) \hat{\theta}_{1}(V(x))+$ $\sigma^{W}(|w|) \hat{\theta}_{2}(W(\kappa, e))-\left(c-1-\varepsilon_{1}-\varepsilon_{2}\right) \gamma^{2} W^{2}(\kappa, e)-\varrho(|x|)-\varrho$ $(W(\kappa, e))+\hat{\sigma}(|w|)$. By the definition of $\theta(\cdot)$ (cf., Assumption 3), we have $\langle\nabla U(\xi), F(\xi, w, v)\rangle \leq-\varrho(|x|)-$ $\varrho(W(\kappa, e))+\sigma^{V}(|w|) \theta(V(x))-\left(c-1-\varepsilon_{1}-\varepsilon_{2}\right) \gamma^{2} W^{2}(\kappa, e)+$ $\sigma^{W}(|w|) \theta\left(\gamma \tilde{\lambda} W^{2}(\kappa, e)\right)+\hat{\sigma}(|w|)$. It follows from the monotonicity of $\theta \in \mathcal{K}$ and the fact that $\phi(\tau) \in\left[\tilde{\lambda}, \tilde{\lambda}^{-1}\right]$ for all $\tau \in\left[0, \tau_{\text {MATI }}\right]$ that $\langle\nabla U(\xi), F(\xi, w, v)\rangle \leq-\varrho(|x|)-\varrho(W(\kappa, e))+$ $\sigma(|w|) \theta(U(\xi))-\left(c-1-\varepsilon_{1}-\varepsilon_{2}\right) \gamma^{2} W^{2}(\kappa, e)+\hat{\sigma}(|w|)$, where $\sigma(\cdot):=\sigma^{V}(\cdot)+\sigma^{W}(\cdot)$. Picking $\varepsilon_{1}$ and $\varepsilon_{2}$ sufficiently small such that $c>1+\varepsilon_{1}+\varepsilon_{2}$, we have

$$
\begin{aligned}
\langle\nabla U(\xi), F(\xi, w, v)\rangle \leq & -\varrho(|x|)-\varrho(W(\kappa, e))+\sigma(|w|) \theta(U(\xi)) \\
& +\hat{\sigma}(|w|)
\end{aligned}
$$

By application of [12, Lemma IV.1] to $\varrho$ on the right-hand side of (36), there exist $\varrho_{1} \in \mathcal{K}_{\infty}$ and $\varrho_{2} \in \mathcal{L}$ such that $\langle\nabla U(\xi), F(\xi, w, v)\rangle \leq$ $-\varrho_{1}(W(\kappa, e)) \varrho_{2}(W(\kappa, e))-\varrho_{1}(|x|) \varrho_{2}(|x|)+\sigma(|w|) \theta(U(\xi))+\hat{\sigma}$ $(|w|)$. From $\varrho_{1} \in \mathcal{K}_{\infty}, \varrho_{2} \in \mathcal{L}$, and (11), we have $\langle\nabla U(\xi), F$ $(\xi, w, v)\rangle \leq-\varrho_{1} \circ \bar{\alpha}_{x}^{-1}(V(x)) \varrho_{2} \circ \underline{\alpha}_{x}^{-1}(V(x))-\varrho_{1}(W(\kappa, e)) \varrho_{2}$ $(W(\kappa, e))+\sigma(|w|) \theta(U(\xi))+\hat{\sigma}(|w|)$. By the monotonicity of $\varrho_{1}$ and $\varrho_{2}$, and the fact that $\phi(\tau) \geq \tilde{\lambda}$ for all $\tau \in\left[0, \tau_{\text {M ATI }}\right]$, there exists a function $\tilde{\varrho} \in \mathcal{P} \mathcal{D}$ such that for almost all $(\xi,(w, v)) \in \mathcal{C}$ the following holds:

$$
\langle\nabla U(\xi), F(\xi, w, v)\rangle \leq-\tilde{\varrho}(U(\xi))+\sigma(|w|) \theta(U(\xi))+\hat{\sigma}(|w|) .
$$

For any $(\xi,(w, v)) \in \mathcal{D}$, we have $U(G(\xi, w, v))=V(x)+$ $\gamma \phi(0) W^{2}(\kappa+1, h(\kappa, e, v))$. According to (9) and (14), and since $\phi(0)=\tilde{\lambda}^{-1}$, it is obtained that

$$
\begin{aligned}
& U(G(\xi, w, v))=V(x)+\gamma \phi(0) W^{2}(\kappa+1, h(\kappa, e, v)) \\
& \leq V(x)+\gamma \tilde{\lambda}^{-1}\left(\lambda^{2} W^{2}(\kappa, e)+2 \lambda W(\kappa, e) \sigma_{v}(|v|)+\sigma_{v}^{2}(|v|)\right) .
\end{aligned}
$$

Using Young's inequality, we have $U(G(\xi, w, v)) \leq V(x)+$ $\gamma \tilde{\lambda}^{-1} \lambda^{2}\left(1+\varepsilon_{d}\right) W^{2}(\kappa, e)+\hat{\sigma}_{v}(|v|)$, where $\hat{\sigma}_{v}(\cdot):=\gamma \tilde{\tilde{\lambda}}^{-1}\left(\frac{1}{\varepsilon_{d}}+\right.$ 1) $\sigma_{v}^{2}(\cdot)$. From the fact that $\tilde{\lambda}>\lambda$, one can take $\varepsilon_{d}$ sufficiently small such that $\lambda^{2}\left(1+\varepsilon_{d}\right)<\tilde{\lambda}^{2}$. Thus

$$
U(G(\xi, w, v)) \leq U(\xi)+\hat{\sigma}_{v}(|v|)
$$

for all $(\xi,(w, v)) \in \mathcal{D}$.

Define a scaling function $\mu: \mathbb{R}_{\geq 0} \rightarrow \mathbb{R}_{\geq 0}$ satisfying

$$
\mu(0)=0, \quad \frac{d \mu}{d s}=\frac{1}{1+\theta(s)} \quad \forall s \in \mathbb{R}_{>0} .
$$

We note that from (16) we have $\mu \in \mathcal{K}_{\infty}$. Now, let $S(\cdot):=\mu(U(\cdot))$. So, for any $(\xi,(w, v)) \in \mathcal{C}$, we have $\langle\nabla S(\xi), F(\xi, w, v)\rangle \leq$ $\frac{1}{1+\theta(U(\xi))}(\sigma(|w|) \theta(U(\xi))-\tilde{\varrho}(U(\xi))+\hat{\sigma}(|w|)) \leq-\bar{\varrho}(U(\xi))+\bar{\sigma}$ $(|w|), \quad$ with $\quad \bar{\sigma}(\cdot):=\sigma(\cdot)+\hat{\sigma}(\cdot) \quad$ and $\quad \bar{\varrho}(\cdot):=\frac{\tilde{\varrho}(\cdot)}{1+\theta(\cdot)}$. Defining $\hat{\varrho}(\cdot):=\bar{\varrho} \circ \mu^{-1}(\cdot)$, we have that

$$
\langle\nabla S(\xi), F(\xi, w, v)\rangle \leq-\hat{\varrho}(S(\xi))+\bar{\sigma}(|w|) .
$$

On the other hand, we have for all $(\xi,(w, v)) \in \mathcal{D}$ that $S(G(\xi, w, v))-S(\xi)=\mu(U(G(\xi, w, v)))-\mu(U(\xi))$. Considering two different cases: when $U(G(\xi, w, v)) \geq U(\xi)$ and when $U(G(\xi, w, v))<U(\xi)$, and using (38), (39), and the monotonicity of $\mu$, we have that

$$
S(G(\xi, w, v)) \leq S(\xi)+\hat{\sigma}_{v}(|v|)
$$

for all $(\xi, w, v) \in \mathcal{D}$. Define the hybrid $\operatorname{arcs} z$ and $\omega$ by

$$
\begin{gathered}
z(t, j):=S(\xi(t, j))-\omega(t, j) \\
\omega(t, j):=\int_{0}^{t} \bar{\sigma}(|w(s, i(s))|) d s+\sum_{\left(t^{\prime}, j^{\prime}\right) \in \Gamma(v)} \hat{\sigma}_{v}\left(\left|v\left(t^{\prime}, j^{\prime}\right)\right|\right) . \\
(0,0) \preceq\left(t^{\prime}, j^{\prime}\right) \prec(t, j)
\end{gathered}
$$

It follows from (40), (42), and (43) that

$$
\dot{z}(t, j) \leq-\hat{\varrho}(S(\xi(t, j)))=-\hat{\varrho}(\max \{z(t, j)+\omega(t, j), 0\})
$$

for all $j \in \mathbb{Z}_{\geq 0}$ and almost all $t$ such that $(t, j) \in E$. From (41)-(43), we have

$$
z\left(t_{j}, j\right) \leq z\left(t_{j}, j-1\right)
$$

for all $\left(t_{j}, j-1\right) \in E$ such that $\left(t_{j}, j\right) \in E$. By application of Lemma 12 to (44) and (45), there exists $\tilde{\beta} \in \mathcal{K} \mathcal{L}$ such that

$$
z(t, j) \leq \max \left\{\tilde{\beta}\left(z_{0}, t\right),|\omega|_{(t, j), \infty}\right\} \leq \tilde{\beta}\left(z_{0}, t\right)+|\omega|_{(t, j), \infty} .
$$

An immediate consequence from (42), (43), and (46) and the fact that $z_{0}=S\left(\xi_{0}\right)$ is that

$$
\begin{gathered}
S(\xi(t, j)) \leq \tilde{\beta}\left(S\left(\xi_{0}\right), t\right)+2 \int_{0}^{t} \bar{\sigma}(|w(s, i(s))|) d s \\
+2 \sum_{\left(t^{\prime}, j^{\prime}\right) \in \Gamma(v)} \hat{\sigma}_{v}\left(\left|v\left(t^{\prime}, j^{\prime}\right)\right|\right) \\
(0,0) \preceq\left(t^{\prime}, j^{\prime}\right) \prec(t, j)
\end{gathered}
$$

for all $(t, j) \in E$. Noting that $t \geq \epsilon j$ for all $(t, j) \in E$ yields

$$
\begin{aligned}
& S(\xi(t, j)) \leq \tilde{\beta}\left(S\left(\xi_{0}\right), 0.5 t+0.5 \epsilon j\right)+2 \int_{0}^{t} \bar{\sigma}(|w(s, i(s))|) d s \\
&+2 \sum_{\left(t^{\prime}, j^{\prime}\right) \in \Gamma(v)} \hat{\sigma}_{v}\left(\left|v\left(t^{\prime}, j^{\prime}\right)\right|\right) \\
&(0,0) \preceq\left(t^{\prime}, j^{\prime}\right) \prec(t, j)
\end{aligned}
$$

for all $(t, j) \in E$. By the definition of the function $S$ and exploiting (34) yields $\mu \circ \underline{\alpha}(|(x(t, j), e(t, j))|) \leq$ $\tilde{\beta}\left(\mu \circ \bar{\alpha}\left(\left|\left(x_{0}, e_{0}\right)\right|\right), 0.5 t+0.5 \epsilon j\right)+2 \int_{0}^{t} \bar{\sigma}(|w(s, i(s))|) d s+$

$2 \sum\left(t^{\prime}, j^{\prime}\right) \in \Gamma(v), \quad \hat{\sigma}_{v}\left(\left|v\left(t^{\prime}, j^{\prime}\right)\right|\right)$. Denoting $\alpha:=\mu \circ \underline{\alpha}$, $(0,0) \preceq\left(t^{\prime}, j^{\prime}\right) \prec(t, j)$

$\beta\left(r, s_{1}, s_{2}\right):=\tilde{\beta}\left(\mu \circ \bar{\alpha}(r), 0.5 s_{1}+0.5 \epsilon s_{2}\right), \quad \eta_{1}(s):=2 \bar{\sigma}(s), \quad$ and $\eta_{2}(s):=2 \hat{\sigma}_{v}(s)$ gives the conclusion. 


\section{REFERENCES}

[1] G. C. Walsh, H. Ye, and L. G. Bushnell, "Stability analysis of networked control systems," IEEE Trans. Control Syst. Technol., vol. 10, no. 3, pp. 438-446, May 2002.

[2] D. Nešić and A. R. Teel, "Input-output stability properties of networked control systems," IEEE Trans. Autom. Control, vol. 49, no. 10, pp. 16501667, Oct. 2004.

[3] W. P. M. H. Heemels and N. van de Wouw, Stability and Stabilization of Networked Control Systems. London, U.K.: Springer, 2010, pp. 203-253.

[4] D. Borgers, R. Geiselhart, and W. Heemels, "Tradeoffs between quality-ofcontrol and quality-of-service in large-scale nonlinear networked control systems," Nonlinear Anal. Hybrid Syst., vol. 23, no. Suppl. C, pp. 142$165,2017$.

[5] S. Heijmans, R. Postoyan, D. Nešić, N. Noroozi, and W. Heemels, "Stability analysis of networked linear control systems with direct-feedthrough terms," Automatica, vol. 96, pp. S186-S200, 2018.

[6] D. Carnevale, A. R. Teel, and D. Nešić, "A Lyapunov proof of an improved maximum allowable transfer interval for networked control systems," IEEE Trans. Autom. Control, vol. 52, no. 5, pp. 892-897, May 2007.

[7] W. P. M. H. Heemels, A. R. Teel, N. van de Wouw, and D. Nešić, "Networked control systems with communication constraints: Tradeoffs between transmission intervals, delays and performance," IEEE Trans. Autom. Control, vol. 55, no. 8, pp. 1781-1796, Aug. 2010

[8] V. Dolk, D. Borgers, and W. Heemels, "Output-based and decentralized dynamic event-triggered control with guaranteed $\mathcal{L}_{p}$-gain performance and Zeno-freeness," IEEE Trans. Autom. Control, vol. 62, no. 1, pp. 34-49, Jan. 2017.

[9] D. Nešić and A. R. Teel, "Input-to-state stability of networked control systems," Automatica, vol. 40, pp. 2121-2128, 2004.

[10] M. Abdelrahim, R. Postoyan, J. Daafouz, and D. Nešić, "Robust eventtriggered output feedback controllers for nonlinear systems," Automatica, vol. 75, pp. 96-108, 2017.

[11] E. D. Sontag, "Comments on integral variants of ISS," Syst. Control Lett., vol. 34 , no. 1 , pp. $93-100,1998$.
[12] D. Angeli, E. D. Sontag, and Y. Wang, "A characterization of integral input-to-state stability," IEEE Trans. Autom. Control, vol. 45, no. 6, pp. 1082-1097, Jun. 2000

[13] C. M. Kellett and F. R. Wirth, "Nonlinear scaling of (i)ISS-Lyapunov functions," IEEE Trans. Autom. Control, vol. 61, no. 4, pp. 1087-1092, Apr. 2016.

[14] R. Postoyan, N. van de Wouw, D. Nesic, and W. P. M. H. Heemels, "Tracking control for nonlinear networked control systems," IEEE Trans. Autom. Control, vol. 59, no. 6, pp. 1539-1554, Jun. 2014.

[15] N. Noroozi, R. Geiselhart, and F. Wirth, "On integral input-to-state stability analysis of networked control systems," IFAC-PapersOnLine, vol. 50, no. 1, pp. 10078-10083, 2017.

[16] H. K. Khalil, Nonlinear Systems, 3rd ed. Englewood Cliffs, NJ, USA: Prentice-Hall, 2002.

[17] C. Cai and A. R. Teel, "Characterizations of input-to-state stability for hybrid systems," Syst. Control Lett., vol. 58, no. 1, pp. 47-53, 2009.

[18] R. Goebel, R. G. Sanfelice, and A. R. Teel, Hybrid Dynamical Systems: Modeling, Stability, and Robustness. Princeton, NJ, USA: Princeton Univ. Press, 2012.

[19] M. Tabbara and D. Nešić, "Input-output stability with input-to-state stable protocols for quantized and networked control systems," in Proc. 47th IEEE Conf. Decis. Control, Cancun, Mexico, 2008, pp. 2680-2685.

[20] N. Noroozi, A. Khayatian, and R. Geiselhart, "A characterization of integral input-to-state stability for hybrid systems," Math. Control Signals Syst., vol. 29, no. 3, p. 13, Jun. 14, 2017, doi: 10.1007/s00498-017-0199-4.

[21] L. Zaccarian, A. R. Teel, and D. Nešić, "On finite gain $\mathcal{L}_{p}$ stability of nonlinear sampled-data systems," Syst. Control Lett., vol. 49, no. 3, pp. 201212, 2003.

[22] W. Liu, Y. Chitour, and E. Sontag, "On finite-gain stabilizability of linear systems subject to input saturation," SIAM J Control Optim., vol. 34, no. 4, pp. 1190-1219, 1996

[23] R. Azouit, A. Chaillet, Y. Chitour, and L. Greco, "Strong iISS for a class of systems under saturated feedback," Automatica, vol. 71, pp. 272-280, 2016. 